\title{
Rectovaginal Fistula Due to an Erosive Pessary: A Case Report
}

\author{
Waleed Inayat Mohamed,' Dorea Emmanuel, ${ }^{2}$ Shahzaib Maqbool, ${ }^{3}$ Ali Asad, ${ }^{4}$ Asad Jawad, ${ }^{5}$ Arham Ihtesham. ${ }^{3}$
}

\begin{abstract}
Background: Pelvic organ prolapse (POP) occurs when pelvic organs protrude through the vagina due to displacement from their normal anatomic position, as a result of a weakening of the pelvic muscles. Pessaries have long been used to treat POP, but they may result in rectovaginal fistulas as a rare complication. The Case: We report a rare case of a rectovaginal fistula following vaginal ring pessary use in an 82 -year-old G $7 \mathrm{P} 7$ woman. She had a past medical history of bipolar disorder, and initially presented for nursing care and psychiatric support. A trained psychologist evaluated her, and found no psychological morbidity. A two-day history of a fall, diffuse abdominal pain, and chronic urinary incontinence were rather identified. Cynecological pelvic examination revealed a rectovaginal fistula and impacted stools in the vagina. Conclusion: The use of ring pessaries, though a conservative management option for POP, may lead to a rectovaginal fistula, a rare but severe complication.
\end{abstract}

Key Words: Pessaries; Rectovaginal fistula; Adverse effects (Source: MeSH-NLM).

\section{Introduction}

Pelvic organ prolapse (POP) is a common finding in obese elderly women, with $3-6 \%$ symptomatic and about $50 \%$ clinical prevalence rates. ${ }^{1-3}$ Treatment options for POP range from non-invasive pelvic floor exercises and medical management to more invasive surgical options. Vaginal pessary insertion is an inexpensive, helpful, and easy-to-use treatment option for POP with a high success rate. ${ }^{4}$ It is the first-line treatment option for women with mild prolapse, frail and elderly women (poor surgical candidates), and women who refuse surgery. ${ }^{5}$ Severe complications of pessary use are rare but include rectovaginal fistula, ${ }^{6-14}$ vesicovaginal fistula, ${ }^{15}$ cervical entrapment, 16 and intestinal obstruction. ${ }^{17}$ Few cases of rectovaginal fistula have been reported in the literature. We report a case of rectovaginal fistula, following the use of a vaginal ring pessary in an 82-year-old woman.

\section{The case}

An 82-year-old G $7 \mathrm{P} 7$ woman with a past medical history of bipolar disorder presented for nursing care and psychiatric support. A trained psychologist evaluated the patient and found no psychological morbidities. However, a two-day history of a fall, diffuse abdominal pain, and chronic urinary incontinence were identified. A thorough neurological exam revealed that the patient was well oriented in time, place, and person. She had slight reduction in power of upper and lower limbs. The patient was unable to move, and complained of back and abdominal pain. An orthopedic consultation was placed to rule out a vertebral fracture as a fall history was associated with reduced mobility. She had stable vital signs. The orthopedic examination was consistent with reduced power (grade 4). An otherwise unremarkable radiograph of the lumbar spine revealed a ring pessary lying vertically and displaced in the rectum (Figure 3).

Gynecological pelvic examination revealed a rectovaginal fistula Impacted stools were present in the vagina; they were manually removed. No complaints of vaginal or rectal bleeding or other

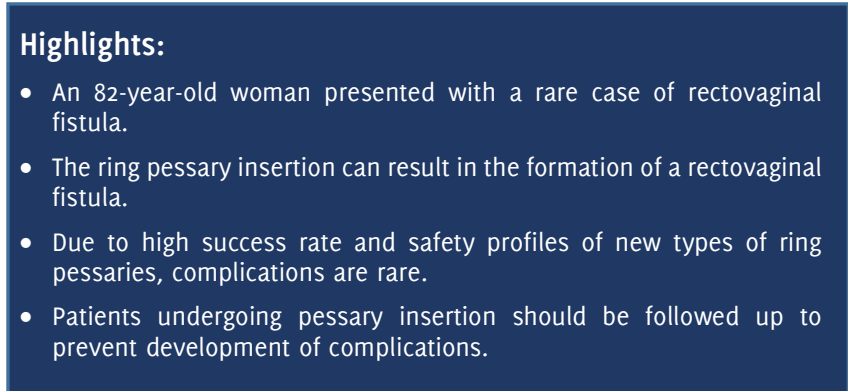

gastrointestinal symptoms were reported. An abdominal/pelvic ultrasonogram was unremarkable. The following day, colonoscopy revealed a low-lying large rectovaginal fistula involving the rectum and anal canal (Figure 1). Two large masses were observed-one at the posterior wall of the vagina and the other attached to the rectal wall at the fistula site. A misplaced ring pessary was removed from the rectum. Impacted stools were extracted from the rectum and vagina. Biopsies of the masses were excised and sent for histopathology examination, which revealed the presence of chronic granulation tissues that resulted in a gradual erosion of the gut mucosa and eventually forming a fistula (Figure 2). Further questioning revealed that the patient had urinary incontinence secondary to POP that occurred two years ago. At the time, she chose a non-invasive treatment of ring pessary insertion. Follow-up was expected, but an enema was never performed due to patient refusal for a detailed examination and enema. The patient was also incompliant towards regular follow-up visits. The pessary improved symptoms of incontinence initially, but became worse gradually. A management plan was devised to mobilize the patient and proceed with an initial dysfunctioning loop colostomy with a definitive plan of fistula repair and colostomy reversal in subsequent surgeries due to the deteriorated condition of the vaginal tissue. The patient underwent the procedure without complications.

\footnotetext{
Bachelor of Medicine/Bachelor of Surgery (MBBS), University of Health Sciences, Pakistan.

2 Bachelor of Medicine/Bachelor of Surgery (MBBS), Awali Hospital, Bahrain.

3 Bachelor of Medicine/Bachelor of Surgery (MBBS), Rawalpindi Medical University, Pakistan.

4 Bachelor of Medicine/Bachelor of Surgery (MBBS), Royal College of Surgeons in Ireland-Medical University of Bahrain (RCSI-MUB).

Bachelor of Medicine/Bachelor of Surgery (MBBS), Doctors' Hospital, Pakistan.
}

About the Author: Shahzaib Maqbool recently graduated from the Rawalpindi Medical University, Pakistan. Currently, I am a House Physician at the Department of Pediatrics of the Holy Family Hospital, Rawalpindi-Pakistan. I have previously worked as a volunteer in a Non-Profit Organization that guides medical students. In addition, I serve on the Medical Student Advisory Board (MSAB) of the International Journal of Clinical Research (IJCR-Central).

Correspondence:

Shahzaib Maqbool

Address: Tipu Rd, Chamanzar Colony, Rawalpindi, Punjab 46000, Pakistan Email: hasanshahzaib299@gmail.com
Editor: Francisco J. Bonilla-Escobar Student Editors: Brandon Belbeck $\circlearrowright$ Adam Dinoff Copyeditor: Johnmark Boachie Proofreader: Leah Komer Layout Editor: Anna-Maria Chantaliyska
Submission: Aug 17, 2021 Revisions required: $\operatorname{Sep} 5,2121$, Oct 10, 2021 Response: Sep 7, 2021, Oct 13,2021 Acceptance: Oct 10, 2021 Publication: 0 ct 27,2021 Publication: Oct 27, 2021 Process: Peer-reviewed 
Figure 1. Colonoscopy Showing Impacted Feces.

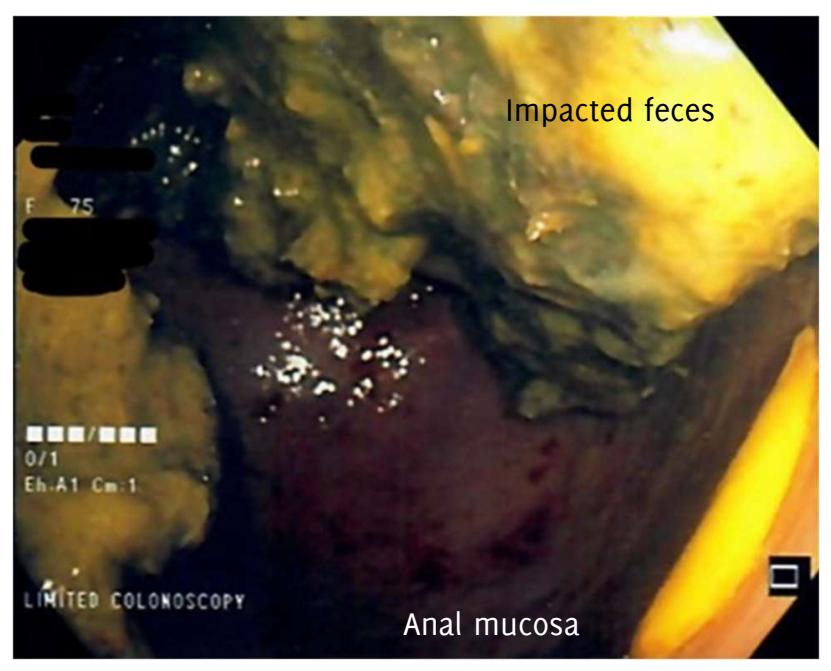

Figure 2. Colonoscopy of Inflamed Mass in Rectovaginal Fistula.

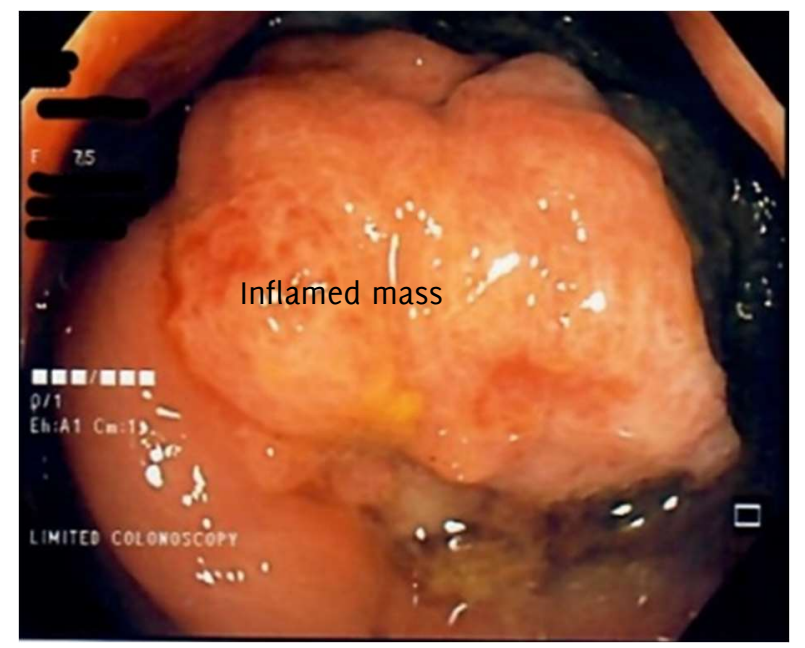

Figure 3. Antero-Posterior View of Pelvic Radiograph Showing Displaced Ring Pessary (Represented by Black Arrow).

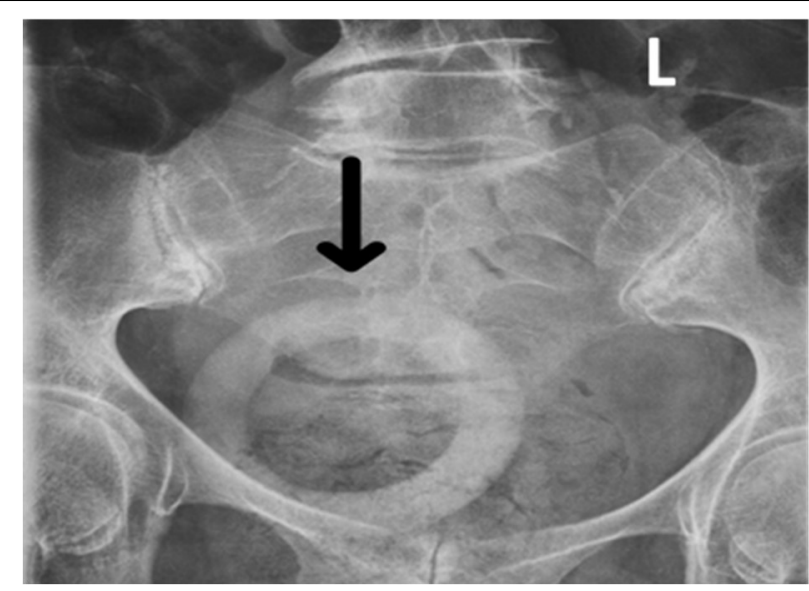

The patient stayed at the hospital for nursing care, and was routinely examined for improvement. Stools were occasionally removed from the vagina and rectum. During her stay, the patient suffered from multiple episodes of urinary tract infections (UTIS), which were treated promptly following positive culture reports. The patient stayed at the hospital for three months due to social reasons. A repeat colonoscopy after three months revealed a reduction in size of the chronic granulation tissue and a persistent fistula. Further pelvic examinations revealed the presence of a persistent Grade 2 cystocele with atrophic vaginal walls without ulceration. The cystocele was reduced manually. A subsequent positron emission tomography (PET) scan, which was performed to rule out suspicious rectal/uterine carcinoma, was negative for the presence of a carcinoma. Posterior bladder wall thickening and osteoporosis were appreciated. Due to old age and patient's choice, closure of fistula and reversal colostomy were called off, and the patient was put on a conservative management plan. She was discharged in a stable condition with the provision of continuous nursing support at home. Stoma care was advised. Clean enemas were provided periodically. The patient was under clinical care for almost four months with strict observation. Psychological support and counselling remained consistent throughout patient stay. The patient's consent was obtained to publish this case report.

\section{Discussion}

POP is a relatively common finding. About $25 \%$ of women in the United States have reported symptoms of pelvic floor disorders, including POP. ${ }^{2}$ Pessaries have been considered an effective treatment method for POP, and have been in use for a long time in different forms, ranging from fruits, metals, cotton, to wool. ${ }^{18}$ With medical advancement, pessaries are mainly composed of silicon currently; other various types exist. $^{5}$ Common complications of pessary use include discharge, bleeding, irritation, and ulceration. ${ }^{18}$ Rare complications, such as rectovaginal fistulas, are now more commonly reported in the literature, particularly in older women after 3-5 years of insertion. ${ }^{6-14}$ Our patient developed a fistula within two years of insertion, even with regular follow-up visits (but rejecting attempts for enema scans during visits), and experienced no significant symptoms. Detailed gynecological pelvic examination, including an enema, should be performed at each visit to assess the condition and location of the pessary. Pelvic radiography may be added. Proper patient selection, physician awareness, and continuous care post-insertion are the factors that play an essential role in the use of pessary devices. Different treatment options have been used in the past to manage rectovaginal fistulas, including a vaginal estradiol cream, ${ }^{12}$ transanal fistula repair, 9 transverse transperineal repair, ${ }^{8}$ and a transvaginal approach. ${ }^{14}$ The management of such cases begins with careful pessary removal and a detailed physical examination, specifically a gynecological pelvic examination, which paves the way for a definitive management plan. In this case, we planned an initial dysfunctioning colostomy (A dysfunctioning stoma is created in the initial surgery as a temporary diverting pathway. It is subsequently closed when the anastomosis fully heals), which was completed uneventfully. Enough time was given for the inflamed mucosa to heal, with a plan to repair the rectovaginal fistula and reverse colostomy subsequently in the future. Three months post-surgery, the granulation tissues had decreased in size. However, on further discussion with the patient, we decided not to proceed with any further surgical treatment, with consideration of the patient's will, frailty, and potential complications related to the procedures. The patient was advised on continuous nursing and stoma care. Six months post-procedure, the stoma was in a good working condition without any complications.

\section{Recommendations}

We recommend the standardization of guidelines on the long-term use of pessaries for POP and treatment of rectovaginal fistula as a complication of pessary use. Regular follow-up after pessary insertion and patient education are essential factors to prevent complications. Patients should be counseled and informed about the rare but serious complication of fistula formation in case of incompliance with followups. The management plan must be tailored towards each patient's need and choice. 


\section{References}

1. Barber MD, Maher C. Epidemiology and outcome assessment of pelvic organ prolapse. Int Urogynecol J. 2013 Nov;24(11):1783-90.

2. Nygaard I, Barber MD, Burgio KL, Kenton K, Meikle S, Schaffer J, Spino C, Whitehead WE, Wu J, Brody DJ; Pelvic Floor Disorders Network. Prevalence of symptomatic pelvic floor disorders in US women. JAMA. 2008 Sep 17;300(11):1311-6.

3. Swift S, Woodman P, O'Boyle A, Kahn M, Valley M, Bland D, Wang W, Schaffer J. Pelvic Organ Support Study (POSST): the distribution, clinical definition, and epidemiologic condition of pelvic organ support defects. Am J Obstet Gynecol. 2005 Mar;192(3):795-806.

4. Clemons JL, Aguilar VC, Tillinghast TA, Jackson ND, Myers DL. Patient satisfaction and changes in prolapse and urinary symptoms in women who were fitted successfully with a pessary for pelvic organ prolapse. Am J Obstet Gynecol. 2004 Apr;190(4):1025-9.

5. Jones $\mathrm{KA}$, Harmanli 0 . Pessary use in pelvic organ prolapse and urinary incontinence. Rev Obstet Gynecol. 2010 Winter;3(1):3-9.

6. Grody $\mathrm{MH}$, Nyirjesy $\mathrm{P}$, Chatwani $\mathrm{A}$. Intravesical foreign body and vesicovaginal fistula: a rare complication of a neglected pessary. Int Urogynecol J Pelvic Floor Dysfunct. 1999;10(6):407-8.

7. Ozuner $\mathrm{G}$, Elagili F, Aytac E. Rectovaginal fistula secondary to an erosive pessary. Tech Coloproctol. 2015 Aug;19(8):491-2.

8. Tarr M, Culbertson S, Lengyel E. Transverse Transperineal Repair of a Pessaryinduced Mid-rectovaginal Fistula, J Pelvic Med Surg. 2008 May;14(3):199-201.
9. Amshel CE, Tripician NR, Marchetti F, Hellinger MD, Sands LR. Pessary-Induced Rectovaginal Fistula. J Pelvic Med Surg. 2005 Jul;11(4):215-8.

10. Powers K, Grigorescu B, Lazarou G, Greston WM, Weber T. Neglected pessary causing a rectovaginal fistula: a case report. J Reprod Med. 2008 Mar;53(3):2357 .

11. Torbey MJ. Large rectovaginal fistula due to a cube pessary despite routine follow-up; but what is 'routine'? J Obstet Gynaecol Res. 2014 Nov;40(11):2162-5.

12. Cichowski S, Rogers RG. Nonsurgical management of a rectovaginal fistula caused by a Gellhorn pessary. Obstet Gynecol. 2013 Aug;122(2 Pt 2):446-449.

13. Hanavadi S, Durham-Hall A, Oke T, Aston N. Forgotten vaginal pessary eroding into rectum. Ann R Coll Surg Engl. 2004 Nov;86(6):W18-9.

14. Reisenauer $C$, Huebner M. Recto-vaginal fistula after pessary therapy. Arch Gynecol Obstet. 2017 May;295(5):1289-1290.

15. Asumpinwong $C$, Leerasiri $P$, Hengrasmee P. Neglected doughnut pessary in the uterine cavity. BMJ Case Rep. 2019 Apr 14;12(4):e228415.

16. Sivasuriya M. Cervical entrapment of a polythene vaginal ring pessary--a clinical curiosity. Aust N Z J Obstet Gynaecol. 1987 May;27(2):168-9.

17. Roberge RJ, Keller C, Garfinkel M. Vaginal pessary-induced mechanical bowel obstruction. J Emerg Med. 2001 May;20(4):367-70.

18. Vierhout ME. The use of pessaries in vaginal prolapse. Eur J Obstet Gynecol Reprod Biol. 2004 Nov 10;117(1):4-9.

\section{Acknowledgments \\ None}

Conflict of Interest Statement at Funding

The Authors have no funding, financial relationships or conflicts of interest to disclose.

Author Contributions

Conceptualization: WIM, DE, SM; Data Curation: WIM; Formal Analysis: DE, SM, AJ; Investigation: SM, AA, Al; Project Administration: DE; Supervision: AJ, Al; Writing - Original Draft Preparation: WIM, AA; Writing - Review ct Editing: Al.

\section{Cite as}

Mohamed WI, Emmanuel D, Maqbool S, Asad A, Jawad A, Ihtesham A. Rectovaginal fistula due to an erosive pessary: A case report. Int J Med Students. 2021 Oct-Dec;9(4):304-6.

This work is licensed under a Creative Commons Attribution 4.0 International License

ISSN 2076-6327

This journal is published by the University Library System, University of Pittsburgh as part of the Digital Publishing Program and is co-sponsored by the University of Pittsburgh Press. 